\title{
THE EFFECT OF SINGLE-DOSE INTRAPERITONEAL BEVACIZUMAB ON PERITONEAL ADHESION FORMATION
}

\author{
Gokce Acun ${ }^{1}$, Hakan Ozdemir $^{2}$, Oguzhan Sunamak ${ }^{2}$, Zehra Unal Ozdemir ${ }^{2}$, Emel Baskan ${ }^{3}$, \\ Mete Yazi ${ }^{4}$, Berna Savas ${ }^{5}$ and Ugur Berberoglu 3 \\ ${ }^{1}$ Department of General Surgery, Beypazari Governmental Hospital, Ankara, Turkey; ${ }^{2}$ Department of Generab Surgery, \\ Haydarpasa Numune Training and Research Hospital, Istanbul, Turkey; ${ }^{3}$ Department of General Surgery, Ankara \\ Oncology Training and Research Hospital, Ankara, Turkey; ${ }^{4}$ Department of General Surgery, Kırıkkale Governmental \\ Hospital, Kırıkkale, Turkey; ${ }^{5}$ Department of Pathology, Ankara Faculty of Medicine, Ankara University, Ankara, Turkey
}

\begin{abstract}
Background: Intra-abdominal adhesions and their complications following abdominal surgery are serious problems, with an incidence of $67-93 \%$. Prevention of peritoneal adhesion formation may eliminate the need for surgical intervention, decreasing complications, morbidity, and cost. Bevacizumab is a recombinant monoclonal antibody which specifically binds vascular endothelial growth factor, an important cytokine in adhesion formation, and neutralizes its biological activity. We developed an experimental model in rats to determine the effect of bevacizumab in preventing adhesion formation and analyzed its effect both micro- and macroscopically. Methods: We used 32 Wistar rats randomly divided into two groups: Group A (control) and Group B (bevacizumab), with 16 rats each. A modified cecum abrasion model was developed; $0.9 \% \mathrm{NaCl}$ solution was administered intraperitoneally to Group A and bevacizumab to Group B. On day 15, adhesion formation was evaluated both macro- and microscopically. Results: Both micro- and macroscopic adhesion grades in Group B were significantly lower than those of control Group A; macroscopic grades were $2.69 \pm 0.95$ and $0.69 \pm 0.8$, and microscopic grades were $2.25 \pm 1.06$ and $0.5 \pm 0.52$ for Groups A and B, respectively. Conclusions: Bevacizumab was effective in preventing intraperitoneal adhesion formation in our study; however, its inhibitory effects on embryogenesis and the hematopoietic, endocrine, and immune systems may limit its clinical use. (REV INVEST CLIN. 2018;70:279-84)
\end{abstract}

Key words: Peritoneal adhesion. Bevacizumab. Vascular endothelial growth factor.

Corresponding author:

*Hakan Ozdemir,

Haydarpasa Numune Training and Research Hospital

Tıbbiye Cd No: 40 Selimiye

Uskudar 34668, Istanbul, Turkey

E-mail: hakanzdmr@yahoo.com

Received for publication: 15-05-2018

Approved for publication: 08-08-2018 doi: $10.24875 / R I C .18002589$ 


\section{INTRODUCTION}

Intra-abdominal adhesions and their complications following abdominal or pelvic surgery are serious problems. The most frequent causes of intra-abdominal adhesions are laparotomies and intra-abdominal infections. These adhesions increase the risk of injury in subsequent intraabdominal and pelvic surgeries ${ }^{1}$. The incidence of adhesion formation following intra-abdominal surgeries is $67-93 \% \%^{2,3}$. A direct relationship between surgical procedures and adhesion formation has been shown in various studies, suggesting that adhesions due to previous surgeries are the most important cause of postoperative intestinal obstructions ${ }^{4}$. There are numerous macrophages, eosinophils, basophils, and mast cells in the intraperitoneal cavity which originate from monocytes in the blood. These cells have a significant amount of plasminogen activators, allowing blood to be collected in the intraperitoneal cavity uncoagulated. Although the fibrinolytic activity of the mesothelium decreases, fibrin and fibrinous adhesion formation increases in trauma, ischemia, and infections. Adhesion formation is a normal process of peritoneal healing. Mechanical trauma, venous stasis, bacterial contamination, and ischemia are some of the main causes of postoperative peritoneal adhesion (PPA) formation ${ }^{5-7}$. A large number of systemic or intraperitoneal medical treatments have been proposed to decrease PPA ${ }^{8,9}$. Vascular endothelial growth factor (VEGF) is not only a potent angiogenic factor but also an important cytokine affecting adhesion formation. Remodeling and fibroblast function in newly formed tissues are the effects of VEGF on the inflammatory process ${ }^{10}$. Bevacizumab is a recombinant monoclonal antibody which specifically binds VEGF and neutralizes its biological activity ${ }^{11}$. It has antiangiogenic and antitumoral effects. An important delay in tumor progression has been observed with bevacizumab, which also decreases VEGF levels to undetectable limits ${ }^{12,13}$. Angiogenesis and adhesion can be blocked by inhibiting VEGF ${ }^{14,15}$. In an experimental model in rats, we used bevacizumab to block intra-abdominal adhesion formation, an important cause of morbidity and mortality, and tried to demonstrate its VEGF neutralization property both micro- and macroscopically.

\section{METHODS}

This experimental study was approved by the Local Ethics Committee on Animal Experiments. A total of 32 adult female Wistar rats with a mean weight of $200 \mathrm{~g}$, fed with standardized rat food, were randomly divided into a control group (Group A) and a bevacizumab group (Group B), of 16 rats each. Surgery was performed after $12 \mathrm{~h}$ of fasting. A combination of intramuscular ketamine hydrochloride $30 \mathrm{mg} / \mathrm{kg}$ (Ketalar Pfizer, Turkey) and $5 \mathrm{mg} /$ kg xylazine (Rompun Bayer, Turkey) was used as anesthesia. A cecum abrasion model was developed, based on the one described by Ignjatovic et al ${ }^{16}$. Following anesthesia, the abdominal skin was shaved and scrubbed with povidone-iodine $10 \%$ solution. Then, $3 \mathrm{~cm}$-long median laparotomy incisions were made. The cecum was located, and 2 $\mathrm{cm}^{2}$ of its wall and $2 \mathrm{~cm} 2$ of the neighboring peritoneum were abraded by scrubbing with gauze until they bled. Bevacizumab $(0.1 \mathrm{~mL}$ of $2.5 \mathrm{mg} / \mathrm{kg}$ bevacizumab diluted to $5 \mathrm{mg} / \mathrm{mL}$ with $0.9 \% \mathrm{NaCl}$ solution) was administered intraperitoneally in Group B, and $0.9 \% \mathrm{NaCl}$ solution of $0.1 \mathrm{~mL}$ volume was administered intraperitoneally in control Group A. The same degree of abrasion was made in all rats, so the drug dose administered did not depend on the degree of abrasion but on the rat's weight. Oral feeding and drinking began freely following the operation. On day 15 , all rats were sacrificed under anesthesia, and relaparotomy was performed. The investigators who sacrificed the rats were blind to bevacizumab or placebo administration. The adhesions formed were evaluated by two surgeons who were also blind to the sample groups. The macroscopic evaluation was based on the Zuhlke Method, ranging from 0 to 4 (Table 1 and Figs. 1 and 2$)^{17}$. Following macroscopic evaluation, the tissues were excised en bloc, including all intestinal adhesions, and then fixed in $10 \%$ formalin solution for histopathological analysis. Hematoxylin and eosin stained samples were evaluated under light microscope by an expert pathologist on gastro intestinal system. Microscopic scoring was done using the method of Ignjatovic et al. (Table 2) $\overline{16}$.

\section{Statistical analysis}

The SPSS statistics 22 program (IBM SPSS, Turkey) was used in statistical analysis. The Shapiro-Wilk test was used to check the normal distribution of parameters. The Mann-Whitney U-test was used to compare the quantitative parameters between the groups which did not show normal distribution. 
Table 1. Zuhlke method for macroscopic evaluation of adhesions.

\begin{tabular}{ll}
\hline Grade & Findings \\
\hline 0 & No adhesion \\
1 & Thin adhesion, membrane-like adhesions which can be separated by blunt dissection easily. There is \\
& no vascularization \\
2 & Strong adhesion which may be separated slightly by blunt dissection but may need sharp dissection \\
& (vascularization begins) \\
4 & Strong adhesion which can only be separated by sharp dissection; marked vascularization is present \\
& Very strong adhesion fixing organs firmly which can only be separated by sharp dissection and high risk \\
\hline
\end{tabular}

Table 2. Ignjatovic et al. score system for microscopic adhesions.

\begin{tabular}{|c|c|c|}
\hline Grade & Findings & $\frac{\overline{0}}{5}$ \\
\hline Grade 0 & Normal, fibrosis absent & E \\
\hline Grade 1 & Focal slight fibroblast activation, minimal vascular proliferation & $\stackrel{2}{\complement}$ \\
\hline Grade 2 & Widespread slight fibroblastic and vascular proliferation & $\stackrel{ \pm}{ \pm}$ \\
\hline Grade 3 & More marked fibrosis, vascular proliferation & $\xi$ \\
\hline Grade 4 & $\begin{array}{l}\text { Widespread, severe fibroblastic proliferation, vascular proliferation, and inflammatory granulation } \\
\text { development }\end{array}$ & tissue \\
\hline
\end{tabular}

Figure 1. Grade 0 macroscopic adhesion in Group B (bevacizumab).

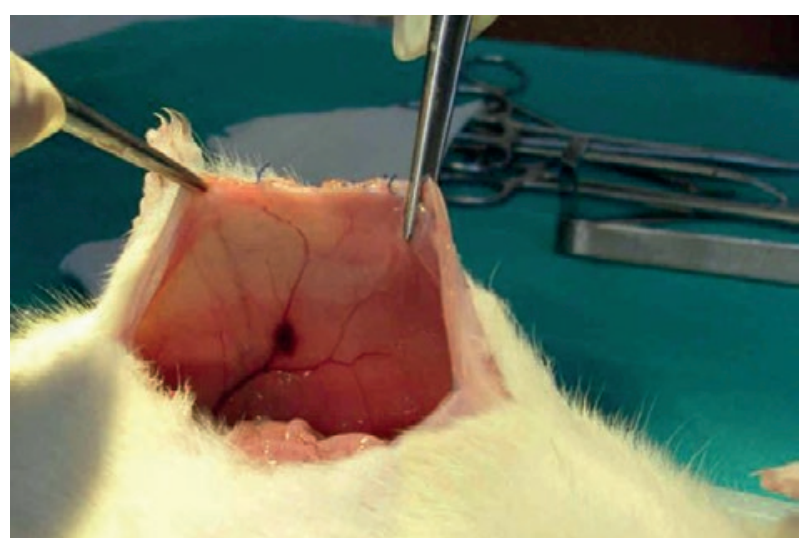

Figure 2. Grade 3 macroscopic adhesion in Group A (control).

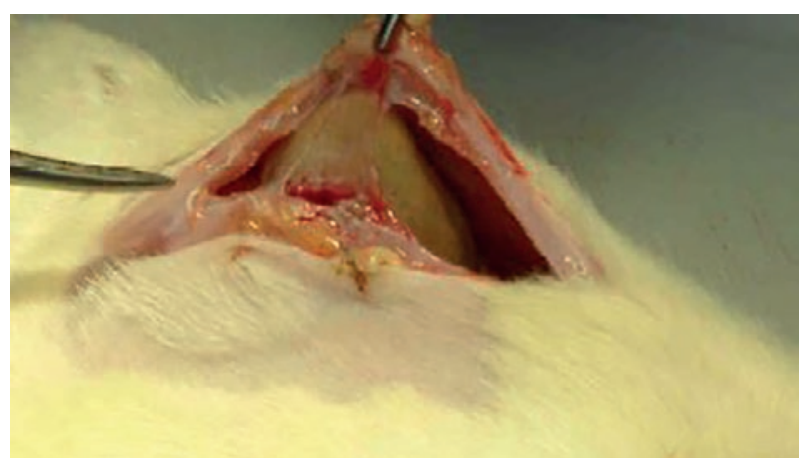

Spearman's rho correlation analysis was used to evaluate the relationship between parameters that did not show normal distribution. Significance level was accepted as $p<0.05$.

\section{RESULTS}

There was no operative or postoperative mortality, wound infection, or wound dehiscence. The groups were analyzed for microscopic and macroscopic adhesions. Both micro- and macroscopic adhesion grades in Group B were lower than those of control Group A (Fig. 3 and Table 3). Macroscopic grades were $2.69 \pm$ 0.95 and $0.69 \pm 0.8$; microscopic grades were $2.25 \pm$ 1.06 and $0.5 \pm 0.52$ for Group A and B, respectively. Micro- and macroscopic grades within each group were evaluated for correlation (Table 4). There was a $73.4 \%$ positive correlation between micro- and macroscopic grades for Group A, which was statistically significant $(p<0.001)$ (Fig. 4). There was an $84.7 \%$ positive correlation between micro- and macroscopic grades for Group B, which was statistically significant $(p<0.001)$ (Fig. 5). 
Figure 3. Macroscopic and microscopic comparison graph of adhesion formation between Group A (control) and B (bevacizumab).

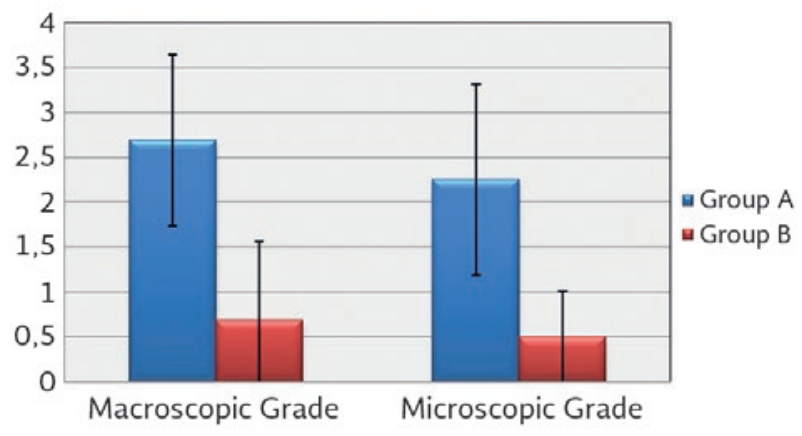

Figure 4. Positive correlation between macroscopic and microscopic grades in Group A (control).

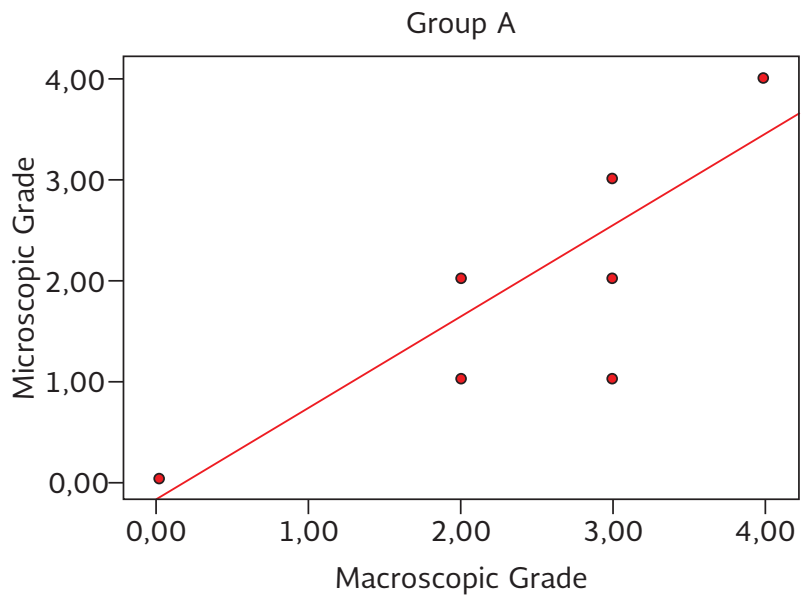

Figure 5. Positive correlation between macroscopic and microscopic grades in Group B (bevacizumab).

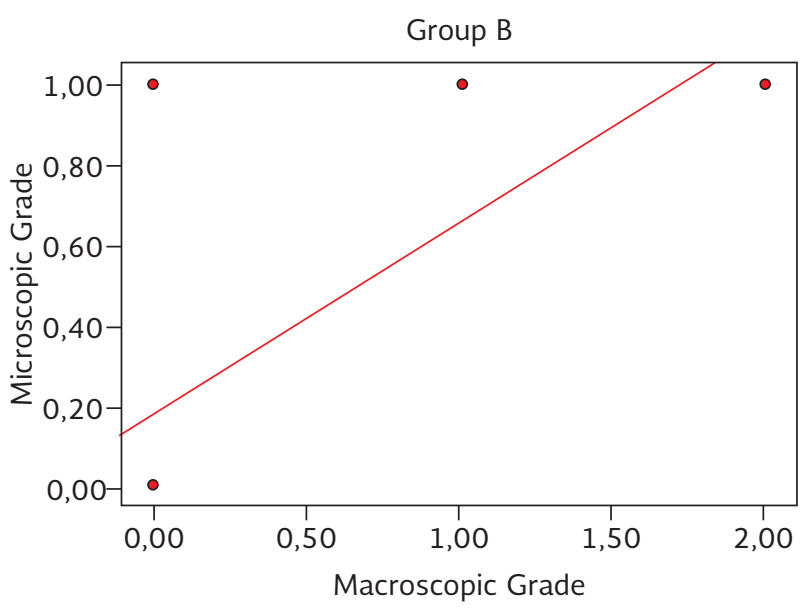

Table 3. Comparison of macroscopic and microscopic grades for each experimental group (Group A, control, and Group B, bevacizumab).

\begin{tabular}{|c|c|c|}
\hline \multirow[t]{2}{*}{ Group } & \multicolumn{2}{|c|}{ Macroscopic grade } \\
\hline & $\mathbf{R}$ & $p$ \\
\hline Group A & & (2) \\
\hline Microscopic grade & 0.734 & $0.001^{*}$ \\
\hline Group B & & $\stackrel{\bar{\Phi}}{c}$ \\
\hline Microscopic grade & 0.847 & $0.000^{*}$ \\
\hline
\end{tabular}

Spearman's rho correlation analysis, ${ }^{*} p<0.05$

Table 4. Comparison of macroscopic and microscopic grades between the experimental groups (Group A, control, and Group B, bevacizumab).

\begin{tabular}{lll|l|l}
\hline Grade & $\begin{array}{c}\text { Group A } \\
(n=16)\end{array}$ & $\begin{array}{c}\text { Group B } \\
(n=16)\end{array}$ & & $p$ \\
\cline { 2 - 3 } & $\begin{array}{c}\text { Median } \\
(\mathrm{Q} 1-\mathrm{Q} 3)\end{array}$ & $\begin{array}{c}\text { Median } \\
(\mathrm{Q} 1-\mathrm{Q} 3)\end{array}$ & & \\
\hline $\begin{array}{l}\text { Macroscopic } \\
\text { grade }\end{array}$ & $3(2-3)$ & $0(0-1.8)$ & $0.000^{*}$ \\
$\begin{array}{l}\text { Microscopic } \\
\text { grade }\end{array}$ & $2(2-3)$ & $0.5(0-1)$ & $0.000^{*}$ \\
\hline
\end{tabular}

Mann-Whitney U-test. ${ }^{*} p<0.05$

\section{DISCUSSION}

Peritoneal adhesions following abdominal operations are results of normal wound healing and constitute a major problem in surgery. Research on methods to prevent post-operative adhesion formation has been an important field for surgeons ${ }^{18,19}$. Factors such as trauma, infections, foreign bodies, and ischemia may cause PPA $^{20}$. Intra-abdominal adhesions may also result in chronic pain, intestinal obstruction, fistula formation, and infertility. A method to prevent peritoneal adhesion formation may eliminate surgical intervention with the associated complications, morbidity, and cost 2 VEGF is an important factor in peritoneal adhesion formation. It plays other roles in various physiological events and etiologies of pathological events within the body, including tumor growth and spread, and the promotion of proliferation, migration, and differentiation of endothelial cells. ${ }^{22}$ Besides its crucial importance in angiogenesis, VEGF also plays a critical role in many endothelial cell functions. VEGF is 
the most specific mitogenic factor known for endothelial cells, plays an important role in vasculogenesis and angiogenesis, and helps tumor growth and hematogenous spread ${ }^{23}$. VEGF is a basic factor in wound healing and is responsible for adhesion formation ${ }^{24}$. Thus, the inhibition of VEGF and neutralization of its effects are important in preventing peritoneal adhesions ${ }^{14,15}$. Bevacizumab is a recombinant IgG1 type monoclonal antibody which binds VEGF and inhibits its biologic activity ${ }^{16}$. It has recently been used intravitreally by ophthalmologists in treating proliferative diseases of the eye without any remarkable toxicity ${ }^{25,26}$. Studies report that inhibiting the biologic activity of VEGF with bevacizumab may limit adhesion formation following intra-abdominal surgery in rats ${ }^{16}$. Our observation that the formation of serious adhesions in all rats in the control group following cecal and serosal abrasions supported the role of serosal injury in adhesion formation. In our study, in spite of making serosal abrasions in the bevacizumab group, the finding of a statistically significant decrease in abrasion formation in both macroscopic and microscopic evaluations may be related to VEGF inhibition. This result suggested that bevacizumab may have been an efficient agent in preventing intraabdominal adhesions. Taking its disadvantages into consideration, the most important adverse effects of bevacizumab are gastrointestinal perforation risk and negative effect on wound healing ${ }^{27}$. Allergy, intestinal bleeding, hypertension, leukopenia, abdominal pain, diarrhea, constipation, nausea, vomiting, and proteinuria are frequently seen side effects of intravenous bevacizumab ${ }^{27}$. We did not observe any prominent side effects in our study, leading us to suspect that these adverse effects might be dose dependent. Angiogenesis during wound healing promotes peritoneal adhesion formation. Inhibition of VEGF, which is responsible for angiogenesis, is promising in the prevention of adhesion formation. Different studies have also shown that bevacizumab was effective in preventing intraperitoneal adhesion formation, both macroscopically and microscopically ${ }^{16,28}$. Recently, intraperitoneal bevacizumab was reported to be well tolerated but not effective in symptom control of gastrointestinal malignancyrelated ascites ${ }^{29}$. They reported that intraperitoneal bevacizumab is safe. The more serious side effects of intravenous bevacizumab were reported in a review $^{30}$. As a result, we showed that intraperitoneal application of bevacizumab was effective in decreasing adhesion formation, which could prevent post-operative adhesion-related intestinat obstructions. However, more randomized prospective clinical studies are needed before bevacizumab is used for this purpose because of its serious side effects.

\section{REFERENCES}

1. ten Broek RP, Bakkum EA, Laarhoven CJ, van Goor $\bar{H}$. Epidemiology and prevention of postsurgical adhesions revisited. Ann Surg. 2016;263:12-9.

2. Liakakos T, Thomakos N, Fine PM, Dervenis C, Young RL. Peritoneal adhesions; etiology, pathophysiology, and clinical significance. Dig Surg. 2001;18:260-73.

3. Morawski B, Nawrot I, Klonowski W, Mądrecki M, Tarnowski W. Peritoneal adhesions as a cause of mechanical small bowel obstruction based on own experience. Pol Przegl Chir. 2015;86: 523-31.

4. Arung $\mathrm{W}$, Meurisse $M$, Detry O. Pathophysiology and prevention of postoperative peritoneal adhesions. World J Gastroenterol. 2011;17:4545-53.

5. Makarchian HR, Kasraianfard A, Ghaderzadeh P, Javadi SM, Ghorbanpoor M. The effectiveness of heparin, platelet-rich plasma (PRP), and silver nanoparticles on prevention of postoperative peritoneal adhesion formation in rats. Acta Cir Bras. 2017;32:22-7

6. Cassidy MR, Sheldon HK, Gainsbury ML, et al. The neurokinin 1 receptor regulates peritoneal fibrinolytic activity and postoperative adhesion formation. J Surg Res. 2014;191:12-8.

7. Giusto G, Beretta G, Vercelli C, et al. A pectin-honey hydrogel prevents postoperative intraperitoneal adhesions in a rat model. BMC Vet Res. 2017;13:55.

8. De Clercq K, Schelfhout C, Bracke M, et al. Genipin-crosslinked gelatin microspheres as a strategy to prevent postsurgical peritoneal adhesions: in vitro and in vivo characterization. Biomaterials. 2016;96:33-46.

9. Poehnert D, Grethe L, Maegel L, et al. Evaluation of the effectiveness of peritoneal adhesion prevention devices in a rat model. Int J Med Sci. 2016;13:524-32.

10. Diamond MP, El-Hammady E, Munkarah A, Bieber EJ, Saed G. Modulation of the expression of vascular endothelial growth factor in human fibroblasts. Fertil Steril. 2005;83:405-9.

11. Kalofonos HP, Grivas PD. Monoclonal antibodies in the management of solid tumors. Curr Top Med Chem. 2006;6: 1687-705.

12. Gordon MS, Margolin K, Talpaz M, et al. Phase 5 safety and pharmacokinetic study of recombinant human anti-vascular endothelial growth factor in patients with advanced cancer. J Clin Oncol. 2001;19:843-50.

13. Hurwitz H, Fehrenbacher L, Novotny W, et al. Bevacizumab plus irinotecan, fluorouracil, and leucovorin for metastatic colorectal cancer. N Engl J Med. 2004;350:2335-42.

14. Chiang SC, Cheng CH, Moulton KS, Kasznica JM, Moulton SL. TNP-470 inhibits intraabdominal adhesion formation. J Pediatr Surg. 2000;35:189-96.

15. Greene AK, Alwayn IP, Nose V, et al. Prevention of intra-abdominal adhesions using the antiangiogenic COX-2 inhibitor celecoxib. Ann Surg. 2005;242:140-6.

16. Ignjatovic D, Aasland K, Pettersen M, et al. Intra- abdominal administration of bevacizumab diminishes intra-peritoneal adhesions. Am J Surg. 2010;200:270-5.

17. Zuhlke HV, Lorenz EM, Straub EM, Savvas V. Pathophysiology and classification of adhesions. Langenbecks Arch Chir Suppl II Verh Dtsch Ges Chir. 1990;1:1009-16.

18. Fredriksson F, Sellberg F, Bowden T, Engstrand T, Berglund D, Lilja HE. Sutures impregnated with carbazate-activated polyvinyl alcohol reduce intraperitoneal adhesions. ] Pediatr Surg. 2017;52:1853-8

19. Brochhausen C, Schmitt VH, Mamilos A,et al. Expression of CD68 positive macrophages in the use of different barrier materials to prevent peritoneal adhesions-an animal study. J Mater Sci Mater Med. 2017;28:15 
20. Koninckx PR, Gomel V. Introduction: quality of pelvic surgery and postoperative adhesions. Fertil Steril. 2016;106:991-3.

21. Conze J, Junge $\mathrm{K}$, Klinge $U$, et al. Intraabdominal adhesion formation of polyprolene mesh. Influence of coverage of omentum and polyglactin. Surg Endosc. 2005;19:798-803.

22. Bikfalvi $A$. Recent developments in the inhibition of angiogenesis: examples from studies on platelet factor-4 and the VEGF/ VEGFR system. Biochem Pharmacol. 2004;68:1017-21.

23. Costache MI, lordache S, Costache CA, Dragos E, Dragos A, Saftoiu A. Molecular analysis of vascular endothelial growth factor (VEGF) receptors in EUS-guided samples obtained from patients with pancreatic adenocarcinoma. J Gastrointestin Liver Dis. 2017;26:51-7.

24. Beddy D, Watson RW, Fitzpatrick JM, O'Connell PR. Increased vascular endothelial growth factor production in fibroblasts isolated from strictures in patients with Crohn's disease. Br J Surg. 2004;91:72-7

25. Henry CR, Sisk RA, Tzu JH, et al. Long-term follow-up of intravitreal bevacizumab for the treatment of pediatric retinal and choroidal diseases. JAAPOS. 2015;19:541-8.
26. Wang JK Su PY, Hsu YR, Chen Y], Chen FT, Tseng YY Comparison of the efficacy of intravitreal aflibercept and bevacizumab for macular edema secondary to branch retinal vein occlusion. J Ophthalmol. 2016;2016:8421940.

27. Gordon MS, Cunningham D. Managing patients treated with bevacizumab combination therapy. Oncology. 2005;69:25-33.

28. Moraloglu $\mathrm{O}$, Issik $\mathrm{H}$, Kilic $\mathrm{S}$, et al. Effect of bevacizumab on postoperative adhesion formation in a rat uterine horn adhesion model and the correlation with vascular endothelial growth factor and Ki-67 immunopositivity. Fertil Steril. 2011; 95:2638-41.

29. Jordan K, Luetkens T, Gog C, et al. Intraperitoneal bevacizumab for control of malignant ascites due to advanced-stage gastrointestinal cancers: a multicentre double-blind, placebocontrolled phase II study-AIO SUP-0108. Eur J Cancer. 2016;63:127-34.

30. Maggiore UL, Bellati F, Ruscito I, et al. Monoclonal antibodies therapies for ovarian cancer. Expert Opin Biol Ther. 2013;13 739-64

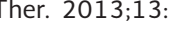

